\section{LAS CARACTERÍSTICAS PERSONALES EN LA EXPERIENCIA DE DOLOR Y EN EL PROCESO DE AFRONTAMIENTO}

\author{
Carmen Ramírez Maestre \\ Universidad de Málaga
}

Resumen Se trata de un trabajo de revisión acerca de cómo una serie de determinantes estables afectan a la experiencia de dolor y a las respuestas del sujeto al mismo. En concreto, se repasa lo que la literatura teórica y empírica dice acerca de cómo las características personales del sujeto con dolor crónico (sexo, edad y variables de personalidad como el neuroticismo, la extraversión y el optimismo disposicional) pueden determinar tanto la percepción de dolor como las estrategias de afrontamiento utilizadas, ejerciendo así un efecto diferencial en la adaptación del sujeto a la situación de dolor crónico. El número de estudios de este tipo con pacientes de dolor crónico es muy escaso. Sin embargo, se contemplan también algunas publicaciones acerca de la influencia de dichas características personales en el ámbito del estrés y del dolor experimental y agudo.

Palabras clave Personalidad, sexo, edad, dolor crónico, afrontamiento.
PERSONALITY TRAITS

IN PAIN EXPERIENCING AND COPING

Abstract The relationships between perceived pain and coping strategies and some stable variables (neuroticism, extroversion, optimism, age, and sex) are analysed. Theoretical and empirical literature about the influence of these personality characteristics on the pain experience and the coping strategies is revised. Although few studies have examined the role of these variables on the chronic pain experience, several investigations on the effects on stress and experimental pain is also reviewed.

Key words Personality, sex, age, chronic pain, coping. 


\section{INTRODUCCIÓN}

El dolor no es una experiencia ajena a ningún ser humano. Sin embargo, se trata de un fenómeno complejo, en tanto que resultado de diversos factores (biológicos, psicológicos y sociales) que interactúan de modo no menos complejo.

Constituye un problema de gran magnitud. De hecho, es una de las causas más comunes por las que los pacientes acuden a consulta médica. Asimismo, no cabe sino analizar los elevados costes económicos que ello supone, costes derivados del uso de medicación, bajas laborales e indemnizaciones (Cruzado, Labrador, De la Puente y Vallejo, 1990). De hecho, autores como Linton (1987), indican que, considerando exclusivamente el dolor de espalda y las lumbalgias, el dolor puede explicar el 25 por 100 de las horas de trabajo perdidas por bajas laborales en países de Europa. Además, hay que considerar que las cifras anteriormente citadas proceden de informes de hospitales, aunque probablemente sólo reflejen la punta del iceberg con respecto a la prevalencia del dolor, ya que hay personas que tratan de manejar su dolor sin buscar atención médica (Turk y Melzack, 1992).

Más allá de estos costes materiales, no se debe olvidar los costes emocionales e interpersonales que el dolor genera en los individuos que lo padecen. Nos referimos a las consecuencias que se derivan del padecimiento de dolor y que afectan a las relaciones interpersonales (mayor dependencia, menor autonomía, disminución de las habilidades interpersonales, mayor aislamiento social), a las relaciones familiares (cambios en los recursos financieros, en la estructura familiar), a las relaciones de pareja (aumento de la dependencia, cambios en las interacciones sexuales, separación y divorcio) así como a otras cuestiones personales (pérdida de control, deterioro de las funciones cognitivas, malestar psicológico, cambios físicos, cambios en la imagen corporal, pérdida de movilidad).

El dolor, por lo tanto, constituye un problema tanto social como individual, en términos de sufrimiento del paciente, el impacto sobre sus familias, el tiempo de trabajo perdido, los gastos médicos, los costes asociados con las indemnizaciones de la incapacidad y la sobreutilización de los servicios del cuidado de la salud (Donker, 1990).

El nacimiento de la algología o disciplina centrada en el estudio de dolor tiene su origen en 1953 con la obra de Bonica (The management of pain), Jefe del Departamento de Anestesiología de la Universidad de Washington, primer presidente de la Asociación Internacional para el Estudio del Dolor y pionero en el estudio del dolor en tanto que comienza a considerar el mismo como un trastorno con entidad propia y no meramente un síntoma de procesos patológicos. De hecho, es este autor el primer fundador de las unidades clínicas de dolor donde ejercían labores anestesiólogos y neurocirujanos (Madrid-Arias, 1987; Miró y Buela-Casal, 1996). En un principio, el dolor era considerado como un fenómeno simple, una sensación resultante de una estimulación nociva. Se consideraba que su intensidad era proporcional a la magnitud de la lesión, producto final de un sistema lineal de transmisión sensorial. Sin embargo, la insuficiencia del modelo médico para la explicación del dolor tiene como consecuencia la consideración de otros fenómenos que trascienden criterios meramente biológicos y fisiológicos, lo que contribuye a acrecentar el interés de otros ámbitos científicos (entre ellos la psicología) en el tema en cuestión.

Hoy día el dolor es concebido como un fenómeno multidimensional, tal y como se refleja en la definición aportada por la International Association for the Study of Pain (IASP, 1986):

“(el dolor) es, incuestionablemente, una sensación en una parte o partes del cuerpo pero, igualmente, se trata siempre de una experiencia perceptiva y subjetiva desagradable y, por tanto, emocional, resultante de un amplio número de factores: biológicos, psicológicos y sociales" (p.217).

Posiblemente sea dicha multidimensionalidad la responsable de que los resultados logrados hasta ahora en el tratamiento del dolor disten de ser satisfactorios. Si se tienen en cuenta las dificultades para establecer bases bioquímicas y neurofisiológicas en la percepción del mismo, así como la ateoricidad con la que se ha producido tradicionalmente el acercamiento a este ámbito de estudio, caracterizado por enfoques eminentemente unidireccionales (Labrador y Vallejo, 1984), se acaba concluyendo que se trata de un campo de investigación donde todavía queda mucho por hacer. Lo que no puede negarse es el hecho de que las variables psicológicas influyen sobre el dolor. Es más, un creciente número de autores sugieren que las variables psicológicas no sólo son importantes en la experiencia de dolor, sino que representan una enorme influencia en la respuesta de los pacientes al tratamiento (Garofalo, 2000). Las líneas de investigación desde la psicología se han centrado principalmente en el dolor crónico. Este tipo de dolor, que se inicia como un dolor agudo, se constituye en un fenómeno rebelde a toda intervención. Tiene una duración prolongada (habitualmente más de seis meses) y en su transcurso se observa en los pacientes altos niveles de ansiedad y depresión, convirtiéndose en un fenómeno con mayor carga afectiva que emocional (Cruzado y cols., 1990).
ANÁLISIS 
Amparados en esta concepción multidimensional del dolor crónico, y tomando como marco teórico referencial el Modelo Procesual del Estrés propuesto por Lazarus y Folkman (1986), se considera el dolor crónico como una experiencia que se constituye en una situación de tensión crónica mantenida, de acuerdo a la terminología utilizada en el ámbito del estudio del estrés (Kanner, Coyne, Schaefer, y Lazarus, 1981). El individuo, para intentar manejar las demandas de la situación estresante, que considera excesivas para los recursos de los que dispone (o cree que dispone), realiza unos esfuerzos cognitivos y comportamentales, lo cual alude al concepto de afrontamiento, según la definición acuñada por Lazarus y Folkman (1986) en dicho modelo. Desde esta concepción, el afrontamiento se refiere a esfuerzos para manejar las demandas, independientemente del éxito de estos esfuerzos. Por tanto, la efectividad de una determinada estrategia de afrontamiento no es una característica inherente a la misma, por lo que los pensamientos y acciones que conforman el afrontamiento deben ser analizados de modo independiente al éxito de sus resultados, entendiendo como efectivo aquel afrontamiento que incrementa la probabilidad de un resultado adaptativo (Lazarus, 1993).

En líneas generales se defiende que la experiencia de dolor vendrá determinada por: 1) la capacidad del sujeto para manejar la situación que es, en si misma, estresante, lo cual alude al concepto de afrontamiento; 2 ) los recursos con los que cuenta para hacerle frente; y 3) las características personales (sexo, edad, variables de personalidad) que, en interacción con las demás, pueden actuar como variables diferenciadoras de la experiencia de dolor, de acuerdo a lo que viene surgiendo dentro de la revisión de la teoría del estrés como proceso (Sánchez Cánovas y Sánchez López, 1994).

En este contexto se mueve el trabajo de revisión que ahora se presenta. Parece pues que se puede hablar de una serie de determinantes estables de la experiencia de dolor y de las respuestas del sujeto al mismo. En concreto, se repasa lo que la literatura teórica y empírica dice acerca de cómo las características personales del sujeto con dolor crónico (sexo, edad y variables de personalidad como el neuroticismo, la extraversión y el optimismo disposicional) pueden determinar tanto la percepción de dolor como las estrategias de afrontamiento utilizadas, ejerciendo así un efecto diferencial en la adaptación del sujeto a la situación de dolor crónico. El número de estudios de este tipo con pacientes de dolor crónico es muy escaso, sin embargo, se contemplará también algunas publicaciones acerca de la influencia de dichas características personales en el ámbito del estrés y del dolor experimental y agudo.

\section{LA EDAD}

El nivel de sufrimiento y dolor en la población de edad mayor ha sido documentado repetidamente. Un análisis epidemiológico sobre el dolor realizado en una muestra de 3097 ancianos pertenecientes a un área rural, reveló que el $86 \%$ había sufrido algún tipo de dolor durante el año anterior al estudio y el 59\% informó de múltiples quejas de dolor. Además se encontró que es menos probable que las personas de más de 85 años de edad informen de dolor (Mobily, Herr, Clark, y Wallace, 1994). Gagliese y Melzack (1997) afirman que, en líneas generales, los estudios epidemiológicos que han intentado identificar un incremento del dolor a medida que aumenta la edad de los sujetos han sido inconsistentes. Estos autores señalan algunas posibles razones explicativas:

- Estos estudios emplean definiciones diferentes de dolor crónico y/o agudo, lo cual complica las comparaciones de sus resultados.

- Suelen asumir que no existen diferencias en la disposición de los sujetos de distinta edad para informar de síntomas dolorosos. En este sentido, Prohaska y colaboradores (1987) afirman que ciertas investigaciones señalan una menor disposición de los adultos mayores a informar de su dolor.

- Es posible que los individuos que participan en estos estudios no definan el dolor del mismo modo que lo hacen los investigadores.

Por otro lado, algunos autores defienden que se producen cambios en la percepción o sensibilidad al dolor a medida que aumenta la edad de los sujetos. En este sentido, Zoob (1978) afirma que se produce una disminución en la sensibilidad al dolor a medida que aumenta la edad. En cambio, Fordyce (1976) defiende que los pacientes de mayor edad se quejan más del dolor que los pacientes jóvenes. Clark y Meehl (1973) plantean que las quejas de dolor están en función de la tendencia a informar de dolor y no en función de la sensibilidad ante su percepción.

De cualquier forma, habremos de distinguir entre los hallazgos de los estudios experimentales y aquellos que se derivan del análisis de pacientes con dolor crónico. Así, algunas de las conclusiones más relevantes de los trabajos con dolor experimental podrían ser:

1. La mayoría de los estudios coinciden en señalar que las personas de mayor edad tienen un umbral 
de dolor superior al de las personas jóvenes (Gagliese y Melzack, 1997; Tucker, Anderws, Ogle, y Davidson, 1989). No obstante, podemos encontrar otros trabajos con resultados opuestos, esto es, a medida que aumenta la edad de las personas disminuye su umbral ante el dolor (Collin y Stone, 1966). Más lejano en el tiempo, el estudio de Birren y colaboradores (1959) no halló diferencias en el umbral de dolor de sujetos con distinta edad.

2. De cualquier forma, lo que parece más claro es que no existen relaciones simples entre la edad y la percepción del dolor. Por el contrario, es necesario tener en consideración diferentes variables que afectan tanto a la percepción como al informe del dolor que realiza el sujeto (Zimbardo, Cohen, Weisenberg, Dwokin y Firestone, 1966). Una de estas variables es la intensidad del estímulo doloroso utilizado en el experimento en cuestión. Así, Harkins y Chapman $(1976,1977)$ encontraron que cuando el estímulo doloroso es de baja intensidad los adultos de mayor edad informan de menos dolor que los jóvenes, mientras que ante un estímulo doloroso más intenso los adultos mayores manifiestan más dolor que los jóvenes.

No obstante, como bien señala Beecher (1965), debemos tener en cuenta las importantes diferencias cualitativas existentes entre el dolor experimental y el dolor clínico. En el contexto del dolor crónico, tal y como sugieren Gagliese y Melzack (1997), la edad debe de tener un efecto en la experiencia total de dolor. Los resultados acerca de las diferencias en función de la edad en sujetos con dolor crónico son igualmente inconsistentes, aunque algunos resultados sugieren que puede existir un cambio en la cualidad pero no en la intensidad percibida de dolor a medida que aumenta la edad. De esta forma, aunque existen evidencias que señalan que una mayoría significativa de personas mayores puede experimentar dolor crónico, este grupo no es representativo en la distribución de pacientes que acuden a las clínicas de dolor. Por otro lado, esto puede estar relacionado con el hecho de que existe una incidencia distinta de determinados tipos de dolor en función de la edad de los pacientes (Unruh, 1996). Así, Harkins y Price (1992) señalan que los dolores de tipo músculo-esqueletal aumentan con la edad, mientras que es muy baja la incidencia de cefaleas y dolores estomacales en los adultos de mayor edad (VonKorff, Dworkin y Kruger, 1988). En el caso de la lumbalgia, el comienzo del dolor se suele cifrar entre los 20-30 años y la más alta prevalencia entre los 40-60 años (Flor y Turk, 1984).
En definitiva, si bien la mayoría de estudios sugiere que existen diferencias en la percepción del dolor experimental, del agudo y del crónico en función de la edad del individuo, los resultados son ciertamente inconsistentes y contradictorios. Por ello se hace relevante la realización de trabajos que arrojen luz en este sentido.

Mayor es el número de publicaciones acerca de las diferencias entre sujetos de distinta edad en la elección de las estrategias de afrontamiento. Estos estudios se mueven en dos perspectivas teóricas diferentes.

Por un lado, la perspectiva del desarrollo (Guttman, 1964, 1974, 1987) defiende que los individuos tienden a evolucionar desde un afrontamiento más activo hacia otro más pasivo en edades superiores. En esta misma línea se sitúa Pfeiffer (1977) cuando afirma que en edades superiores se produce un incremento en el uso de mecanismos de afrontamiento primitivos y regresivos. De modo que, aunque algunas personas continúan utilizando una gran variedad de mecanismos de afrontamiento adaptativos en edades intermedias y superiores, la mayoría vuelve a utilizar estrategias más primitivas como la negación, la somatización o la proyección.

De otro lado, la perspectiva situacional (Vaillant, $1976,1977)$ se centra en un contexto socioambiental del proceso de estrés-afrontamiento. De este modo, la edad estará asociada con un mayor uso de mecanismos de afrontamiento como la supresión, la anticipación y el humor, además de reducirse el uso de estrategias más inmaduras como la negación o la represión. Los estudios longitudinales de Vaillant $(1976,1977)$ permiten concluir que el uso de estrategias de afrontamiento que posibilitan una mejor adaptación del sujeto se incrementan desde los 20 años hasta la mitad de la edad adulta, de modo que los mecanismos de afrontamiento maduran con la edad. Todavía desde esta perspectiva y sin embargo, en una línea bastante distinta, Costa y McCrae (1993; Costa, Zonderman y McCrae, 1991; McCrae y Costa, 1990) defienden que las estrategias de afrontamiento utilizadas por un individuo apenas cambian a lo largo de su vida adulta. Así, aquellos individuos competentes para manejar los eventos vitales diarios en edades tempranas también son capaces de seguir manejándolos de modo competente en edades superiores. De igual forma, aquellos sujetos que exhiben deficiencias en los mecanismos de afrontamiento en edades tempranas es probable que continúen con los mismos problemas en edades superiores si no se ha intervenido sobre ellos.

Por último, se deben señalar los planteamientos teóricos de Lazarus (Lazarus y Folkman, 1986) y el papel que otorga a la variable edad en su Modelo. Según Folkman, Lazarus, Pimley y Novacek (1987), en general, la
ANÁLISIS 
TABLA 1

ESTUDIOS QUE ANALIZAN LAS DIFERENCIAS, EN FUNCIÓN DE LA EDAD, EN LAS ESTRATEGIAS UTILIZADAS PARA AFRONTAR UNA SITUACIÓN ESTRESANTE

\begin{tabular}{ll}
\hline Autores & Resultados \\
\hline Pearlin y Schooler (1978) & -Los sujetos de mayor edad emplean más \\
& estrategias de afrontamiento basadas en la \\
& reflexión y menos estrategias relacionadas con \\
& la búsqueda de apoyo o consejo.
\end{tabular}

Backman y Molander - Los adultos mayores y de mediana edad (1986a, 1986b y 1991) emplean estrategias de afrontamiento con peores resultados que los jóvenes.

Irion y Blanchard-Fields (1987) -Los jóvenes emplean más estrategias de afrontamiento centradas en la emoción. - Los sujetos de mayor edad que evalúan la situación como controlable emplean más estrategias de afrontamiento centradas en el problema, mientras que si evalúan la situación como incontrolable utilizan estrategias centradas en la emoción.

\begin{tabular}{ll}
\hline Cornelius y Caspi (1987) & $\begin{array}{l}\text {-Los sujetos mayores muestran más habilidad } \\
\text { para manejar la situación que los jóvenes. }\end{array}$ \\
\hline Feifel, Strack y Nagy (1987) & $\begin{array}{l}\text {-No encuentran diferencias en las estrategias } \\
\text { de afrontamiento utilizadas por sujetos de } \\
\text { distinta edad. }\end{array}$ \\
\hline
\end{tabular}

investigación ha mostrado que los adultos jóvenes prefieren utilizar estrategias de afrontamiento dirigidas al problema como la confrontación, la planificación y la búsqueda de apoyo social. En contraste, los adultos mayores prefieren las formas de afrontamiento centradas en la emoción como el distanciamiento, la aceptación de responsabilidad y la reinterpretación positiva. Sin embargo, desde el Modelo Procesual del Estrés se postula que aunque la edad debe influir sobre la evaluación cognitiva de la situación estresante, y de este modo en la elección de las estrategias de afrontamiento, no es uno de los determinantes principales de la conducta de adaptación o de la efectividad del afrontamiento.

Sea cual sea la perspectiva teórica de partida, los resultados de las investigaciones centradas en la relación de la edad con las estrategias de afrontamiento utilizadas por sujetos sometidos a situaciones estresantes son realmente contradictorios. De esta forma, mientras ciertos estudios señalan que los adultos mayores afrontan sus problemas de un modo más adaptativo que los jóvenes (Cornelius y Caspi, 1987; Irion y Blanchard-Fields, 1987; Pearlin y Schooler, 1978), otros arrojan resultados contrarios, según los cuales son los adultos jóvenes los que utilizan estrategias de afrontamiento más eficaces ante el estrés (Backman y Molander, 1986a, 1986b y 1991). Por último, otro grupo de investigadores no encuentra diferencias en los modos de afrontamiento al considerar la edad de los sujetos (Feifel, Strack y Nagy, 1987). En términos generales, las conclusiones de los diversos trabajos sugieren que es más frecuente encontrar diferencias en las estrategias de afrontamiento empleadas por personas de diferentes edades que no encontrarlas. No obstante, existe una falta de claridad con respecto a la dirección de las asociaciones entre la edad y el afrontamiento, en parte debida a las diferentes medidas utilizadas en las investigaciones, así como a la gran variedad de situaciones estresantes consideradas (ver Tabla 1).

Centrados ya en el dolor crónico como la situación estresante a la que los sujetos deben de hacer frente, los datos muestran la misma contradicción. Por un lado, algunos autores encuentran diferencias en las estrategias de afrontamiento empleadas por los adultos mayores y jóvenes. No obstante, mientras unos señalan que los de más edad utilizan en mayor medida estrategias pasivas o centradas en la emoción (Felton y Revenson, 1987; Klinger y Spaulding, 1998), otros concluyen que las estrategias utilizadas por los pacientes mayores son más activas o centradas en el problema que las empleadas por los jóvenes (Prohasha, Leventhal, Leventhal y Keller, 1985). Finalmente, un tercer grupo de estudios no encuentra ninguna diferencia en las estrategias utilizadas en función de la edad de los pacientes (Keefe y Williams, 1990; McCrae, 1982, Soriano y Monsalve, 1999) (ver tabla 2).

Por tanto, aún cuando se cuenta con un número relativamente amplio de trabajos que estudian las diferencias en función de la edad en las estrategias de afrontamiento utilizadas por los sujetos sometidos a situación de estrés, los resultados de los mismos no aclaran el sentido de esas diferencias, ni siquiera coinciden plenamente en la existencia de las mismas. Todo lo cual acentúa la importancia de continuar con la investigación al respecto.

\section{EL SEXO}

En lo referido al papel del sexo en la percepción del dolor, los resultados obtenidos hasta la fecha señalan que pueden existir diferencias en el dolor informado por hombres y mujeres, tanto en el agudo como en el crónico.

Los resultados obtenidos en situaciones de dolor experimental indican que los hombres tienen mayor tolerancia al dolor que las mujeres, mientras que no existen diferencias en el umbral (Rollman y Harris, 1987). Existe además un acuerdo general en que hombres y mujeres difieren en sus respuestas a estímulos dolorosos. Algunos autores señalan que las mujeres manifiestan niveles más elevados de dolor que los hombres en nive- 
TABLA 2

\section{ESTUDIOS QUE ANALIZAN LAS DIFERENCIAS EN FUNCIÓN DE LA EDAD, EN LAS ESTRATEGIAS UTILIZADAS PARA AFRONTAR EL DOLOR CRÓNICO}

\begin{tabular}{ll}
\hline Autores & Resultados \\
\hline Mc Rae (1982) & $\begin{array}{l}\text {-No se encontraron diferencias en la utilización } \\
\text { de las estrategias de afrontamiento del dolor en } \\
\text { pacientes con distintos rangos de edad. }\end{array}$ \\
\hline Prohaska et al (1985) & $\begin{array}{l}\text {-Los pacientes de mayor edad afrontan el } \\
\text { dolor de un modo más activo que los jóvenes. }\end{array}$ \\
\hline Felton y Revenson (1987) & $\begin{array}{l}\text {-Los pacientes mayores utilizan en mayor } \\
\text { medida las estrategias de afrontamiento } \\
\text { pasivas. }\end{array}$ \\
\hline Keefe y Williams (1990) & $\begin{array}{l}\text {-No encontraron diferencias en las estrategias } \\
\text { de afrontamiento utilizadas por pacientes de } \\
\text { distinta edad. }\end{array}$ \\
\hline Sorkin et al (1990) & $\begin{array}{l}\text {-Hallaron más similitudes que diferencias: los } \\
\text { pacientes mayores utilizan más estrategias } \\
\text { cognitivas que los jóvenes. }\end{array}$ \\
\hline Klinger y Spaulding (1998) & $\begin{array}{l}\text {-Los pacientes mayores utilizaron menos } \\
\text { estrategias cognitivas. } \\
\text {-Los pacientes mayores utilizaron menos } \\
\text { estrategias activas o centradas en el problema } \\
\text { tales como la "búsqueda de información", o la } \\
\text { "expresión emocional". }\end{array}$ \\
\hline Soriano y Monsalve (1999) & -No encuentran diferencias. \\
\hline Ramírez-Maestre y cols. (2000) & $\begin{array}{l}\text {-Menor uso de las estrategias activas por parte } \\
\text { de los pacientes de mayor edad. }\end{array}$ \\
\hline
\end{tabular}

les altos de estimulación (Ellermeier y Wetphal, 1995; Faucett, Gordon y Levine, 1994). En este sentido, la investigación llevada a cabo por Feine, Bushnell, Miren y Duncan (1991) señala que las mujeres evalúan el estímulo doloroso como más intenso que los hombres. Además, las mujeres discriminan mejor entre las diferentes intensidades del estímulo. Los autores concluyen que esas diferencias en la discriminación nociceptiva entre hombres y mujeres son indicativas de la existencia de una variación en la percepción del dolor según el sexo del sujeto, de modo que esta diferencia perceptiva se relacionaría más con factores sensoriales que con factores actitudinales o emocionales.

Ruda (1993) postula que existen diferencias en función del sexo en dolor y analgesia. Se cree que las diferencias entre hombres y mujeres influyen en la mediación neuroquímica de la analgesia, de modo que se otorga un papel importante a las variaciones hormonales para modular los mecanismos neuronales de la analgesia. Por otro lado, este mismo autor señala la distinta prevalencia de los tipos de dolor en hombres y mujeres. Así, las mujeres suelen sufrir con más frecuencia que los hombres dolores de cabeza y artritis reumatoide. De hecho, si consideramos un informe del National Health
Interview Study (Lipton, Ship y Larach-Robinson, en prensa), éste proporciona evidencias sobre las diferencias de sexo en dolor orofacial, de modo que, mientras sólo un $20-30 \%$ de los hombres manifestó un dolor de este tipo, el 70-80\% de las mujeres manifestaron haber experimentado dolor en la mandíbula o en la cara durante los 6 meses precedentes al estudio. En consonancia con esto, algunos de los resultados obtenidos en trabajos acerca del dolor crónico revelan que los dolores de cabe$\mathrm{za}$, abdominales y faciales tienen mayor prevalencia en mujeres que en hombres (Vonkorff, Dworkin, y Kruger, 1988). En el caso del dolor de cabeza, el $75 \%$ de las personas que acuden a consulta son mujeres (Olensen, 1978), mientras que en la disfunción temporomandibular la proporción de mujeres a hombres es de 3 a 1 en la clínica (Helkimo, 1979). En definitiva, ciertos investigadores observan una mayor incidencia de problemas de dolor en general en mujeres que en hombres (Crooke, Rideout y Browne, 1984; Margolis, Zimmy, Miller y Taylor, 1984).

Por último, y centrándonos en lo referido a las diferencias en la intensidad de dolor percibido por hombres y mujeres con dolor crónico, los resultados de diferentes estudios son contradictorios. Mientras unos concluyen que las mujeres manifiestan un nivel superior de intensidad de dolor que los hombres (Affleck, Tennen, Keefe, Lefevre, y cols., 1999; Ramírez-Maestre, López y Anarte, 1999), otros no encuentran diferencias en este sentido (Turk y Okifuji, 1999). Es por esto que se puede afirmar que no existen hasta la fecha conclusiones claras y concluyentes acerca de la dirección del efecto de la variable sexo sobre la percepción de dolor de pacientes con dolor crónico, aunque sí parece clara la existencia de diferencias.

La variable sexo también ha sido estudiada por su posible influencia moduladora del afrontamiento al estrés. En este sentido se han realizado dos tipos de análisis. Por un lado, se han estudiado las diferencias en la utilización de ciertas estrategias de afrontamiento en situaciones específicas. En general, los hombres tienden a emplear más estrategias de evitación, mientras que las mujeres utilizan con más frecuencia estrategias de vigilancia (Krohne, Schumacher y Egloff, 1992; Miller y Kirsch, 1987; Weidner y Collins, 1993). Se piensa que aunque un mayor uso de estrategias de evitación parece ser más adaptativo cuando nos enfrentamos a estresores de corta duración, la vigilancia puede ser más adaptativa en situaciones de estrés grave o de larga duración (Weidner y Collins, 1993). Por tanto, cabe pensar que las mujeres se adaptan mejor ante estresores crónicos.

El segundo tipo de análisis sugiere que el sexo se puede considerar una variable moduladora entre el afrontamiento y los resultados consecuentes. Se piensa
ANÁLISIS 
TABLA 3 ESTUDIOS QUE ANALIZAN LAS DIFERENCIAS EN FUNCIÓN DEL SEXO,
EN LAS ESTRATEGIAS UTILIZADAS PARA AFRONTAR UNA SITUACIÓN ESTRESANTE

\begin{tabular}{|c|c|}
\hline Autores & Resultados \\
\hline Quayhagen y Quayhagen (1982) & $\begin{array}{l}\text {-Las mujeres emplean más estrategias de } \\
\text { búsqueda de apoyo que los hombres. }\end{array}$ \\
\hline Labouvie-Vief et al (1987) & $\begin{array}{l}\text {-Las mujeres utilizan más estrategias de } \\
\text { búsqueda de apoyo social y de evitación que } \\
\text { los hombres. } \\
\text {-Los hombres emplean más estrategias } \\
\text { basadas en la solución de problemas. }\end{array}$ \\
\hline $\begin{array}{l}\text { Krohne, Schumaker y Egloff } \\
\text { (1992) / Miller y Kirsch (1987) } \\
\text { Weidmer y Collins (1993) }\end{array}$ & $\begin{array}{l}\text {-Los hombres emplean más estrategias de } \\
\text { evitación y las mujeres de vigilancia. }\end{array}$ \\
\hline Schwartz y Stone (1993) & $\begin{array}{l}\text {-Las mujeres emplean más que los hombres } \\
\text { estrategias de afrontamiento como la } \\
\text { distracción, la relajación y la búsqueda de } \\
\text { apoyo social. }\end{array}$ \\
\hline Porter y Stone (1995) & $\begin{array}{l}\text {-Las mujeres emplean más que los hombres } \\
\text { estrategias de afrontamiento como la } \\
\text { distracción, la relajación y la búsqueda de } \\
\text { apoyo social y la religión. Utilizan más las } \\
\text { estrategias centradas en la emoción. }\end{array}$ \\
\hline
\end{tabular}

que una misma estrategia puede conducir a resultados diferentes en hombres y en mujeres, de modo que estas diferencias dependen de la intención que tiene el sujeto al emplear unas estrategias y no otras (Krohne, 1996). En este sentido, Slangen, Krohne, Stellrecht, y Kleeman (1993) observaron que el uso de la "reestructuración positiva", como estrategia de afrontamiento, tiene buenos resultados en las mujeres y negativos en los hombres. Parece que, mientras los hombres utilizan esta estrategia para reprimir o negar los aspectos amenazantes de la situación, las mujeres emplean la "reestructuración positiva" como una forma de desarrollar unas habilidades cognitivas necesarias para afrontar con éxito el estresor al que se enfrentan.

En definitiva, los resultados de diferentes trabajos empíricos en este sentido son poco concluyentes y a veces contradictorios. Sin embargo, algunos estudios revelan diferencias claras en las estrategias de afrontamiento utilizadas por hombres y mujeres. Así, parece que las mujeres utilizan en mayor medida la búsqueda de apoyo social y estrategias centradas en la emoción, la evitación y la relajación (Labouvie-Vief, Hakim-Larson y Hobart, 1987; Porter y Stone, 1995; Quayhagen y Quayhagen, 1982; Schwartz y Stone, 1993). En cambio, los hombres suelen emplear más estrategias centradas en el problema, la negación, la reinterpretación y la disminución de ciertas actividades que producen tensión (Astor-Dubin y Hammen, 1984; Brems y Johnson,
1989; Holm, Holroyd, Hursey y Penzien, 1986; Stone y Neale, 1984; Verbrugge, 1985; Vingerhoets y Van Heck, 1990) (ver tabla 3).

En el contexto del dolor crónico la situación es muy similar. En algunos estudios los resultados obtenidos señalan que las mujeres hacen un mayor uso de estrategias pasivas (Ramírez-Maestre y cols., 1999) como el catastrofismo (Jensen, Turner, Romano y Lawler, 1994; Reid, Gilbert, C.A. y McGrath, 1994) y la búsqueda de apoyo social (Buckelew, Shutty, Hewett, Landon, y cols., 1990), así como estrategias centradas en la emoción (Affleck y cols., 1999). Si bien Keefe y Williams (1990) no encontraron diferencias entre hombres y mujeres en las estrategias de afrontamiento utilizadas, otras investigaciones ponen de manifiesto que las relaciones entre el sexo y el afrontamiento al dolor crónico son complejas y pueden verse influidas por otras variables (Jensen y cols., 1994), siendo éste el motivo de que a veces no se hallen dichas diferencias (Unruh, 1996). Como señalan los psicólogos del desarrollo, las conductas socialmente aceptadas en hombres y mujeres son muy diferentes. Así pues, cabe preguntarse si en el proceso de socialización no se crean respuestas adecuadas al dolor según el sexo, cobrando entonces importancia el estudio de las diferencias en el afrontamiento del dolor crónico entre hombres y mujeres. Si realmente, como parece, existen diferencias, la cuestión a dilucidar es si éstas son propias del sexo del individuo o fruto del proceso de socialización diferencial (Ellermeier y Westphal, 1995). (ver tabla 4).

TABLA 4

ESTUDIOS QUE ANALIZAN LAS DIFERENCIAS EN FUNCIÓN DEL SEXO, EN LAS ESTRATEGIAS UTILIZADAS PARA AFRONTAR EL DOLOR CRÓNICO

\begin{tabular}{ll}
\hline Autores & Resultados \\
\hline Keefe y Williams (1990) & $\begin{array}{l}\text {-No encontraron diferencias entre hombres y } \\
\text { mujeres en las estrategias utilizadas para } \\
\text { afrontar el dolor crónico. }\end{array}$ \\
\hline Buckelem et al (1990) & $\begin{array}{l}\text {-Las mujeres utilizan en mayor medida la } \\
\text { búsqueda de apoyo social para afrontar el } \\
\text { dolor. }\end{array}$ \\
\hline Jensen et al (1994) & $\begin{array}{l}\text {-Las mujeres hacen mayor uso del } \\
\text { Reid, Gillbert y McGrath (1994) }\end{array}$ \\
\hline Affleck et al (1999) & $\begin{array}{l}\text {-Mayor uso de las estrategias centradas en la } \\
\text { emoción por parte de las mujeres. }\end{array}$ \\
\hline Ramírez-Maestre y cols. (1999) & $\begin{array}{l}\text {-Mayor uso de estrategias pasivas por parte de } \\
\text { las mujeres. }\end{array}$ \\
\hline Ramírez-Maestre y cols. (2000) & $\begin{array}{l}\text {-Mayor uso de las estrategias activas por parte } \\
\text { de los hombres. }\end{array}$ \\
\hline
\end{tabular}




\section{LAS DIMENSIONES DE PERSONALIDAD}

Varios autores defienden que el análisis de la relación existente entre las estrategias de afrontamiento y las variables de personalidad, ayuda a comprender y explicar por qué ciertos factores de personalidad se relacionan a su vez con el nivel de adaptación de los sujetos sometidos a situaciones estresantes (David y Suls, 1999; DeJong, Van Sonderen y Emmelkamp, 1999; Hewitt y Flett, 1996; Lazarus y Folkman, 1986; Martin, Bradley, Alexander, Alarcón, Triana-Alexander, Aaron, y Alberts, 1996). Si bien Lazarus y Folkman (1986) estiman que el neuroticismo y la extraversión no son muy útiles como predictores, McCrae y Costa (1986) establecen que los modos preferidos de afrontamiento derivan de estas dimensiones de personalidad. Desde esta perspectiva, se propone un modelo acerca de la relación entre ambas variables (Hewitt y Flett, 1996). Este modelo asume que la personalidad determina las estrategias de afrontamiento que utilizará el individuo en situaciones estresantes siendo ésas a su vez, las que permitirán al sujeto un alto o bajo nivel de adaptación (Hewitt y Flett, 1996). Los estudios que se enmarcan dentro de este modelo evalúan la posible relación entre los niveles de una determinada variable disposicional de personalidad y la utilización de determinadas estrategias de afrontamiento (Endler y Parker, 1990). En este sentido, la mayoría de las investigaciones se ha centrado en las dimensiones de personalidad más clásicas: el neuroticismo y la extraversión (McCrae y Costa, 1986; Sánchez Cánovas y Sánchez López, 1994).

\section{NEUROTICISMO Y EXTRAVERSIÓN}

Los resultados de varias investigaciones muestran la existencia de una relación significativa entre altos niveles de neuroticismo y el uso de estrategias de afrontamiento que predicen una adaptación deficiente (Asghari, 1997; BenDebba, Torgerson, y Long, 1997; Bolger, 1990; Costa y McCrae, 1990; Endler y Parker, 1990; Epstein y Meier, 1989; Gunthert, Cohen y Armeli, 1999; Jelicic, Kempen y Passchier, 1998; Kempen, Jelicic y Ormel, 1997; McCrae y Costa, 1986; Medvedova, 1998; Morasso, Costantini, Baracco, Borreani y Capelli, 1996; Tschannen, 1996), mientras que altos niveles de extraversión se relacionan con la utilización de estrategias que predicen una mejor adaptación del sujeto (Gómez, Holmberg, Bounds, Fullarton y Gómez, 1999; McCrae y Costa, 1986; Medvedova, 1999; Morasso y cols, 1996; Rim, 1987; Sánchez Cánovas y Sánchez López, 1994). Así, se encuentra evidencia empírica que apoya la teoría según la cual los sujetos con altas puntuaciones en neuroticismo utilizan estrategias de afrontamiento poco eficaces, como el catastrofismo, en el manejo del estrés (Affleck, Tennen, Urrows y Higgins, 1992). De hecho, parece que el uso de este tipo de estrategias de afrontamiento modula la relación entre el neuroticismo y el malestar psicológico (Bolger, 1990). Por otro lado, la extraversión se relaciona con formas activas, sociales y optimistas de manejar el estrés (Costa, Somerfield y McCrae, 1996).

En el ámbito del dolor crónico concebido como situación estresante, según Affleck y colaboradores (1992), el catastrofismo, como estrategia de afrontamiento poco eficaz, media en la relación entre el neuroticismo y la intensidad del dolor percibida. Sin embargo, los resultados del trabajo de Wade, Dougherty, Hart y Rafii (1992b) indican que los niveles de neuroticismo no afectan a la intensidad percibida de dolor, aunque los pacientes con dolor crónico que presentan altos niveles de neuroticismo sí manifiestan un mayor nivel de malestar subjetivo relacionado con el dolor. Por otro lado, existe un grupo de estudios cuyos resultados apuntan hacia una relación directa entre el nivel de neuroticismo y el de dolor (Lauver y Johnson, 1997; Lefebvre, 1998). En la actualidad, los estudios acerca de la relación existente entre los rasgos de personalidad y el grado de adaptación de los pacientes con dolor crónico se centran principalmente en el análisis de la interacción entre neuroticismo y estrategias de afrontamiento (Asghari y Nicholas, 1999). En esta línea, varios estudios encuentran relaciones significativas entre el neuroticismo y ciertas variables relevantes en el ámbito del dolor crónico (Ramírez-Maestre, Valdivia, Esteve y López, 2000). Así, Wade, Dougherty, Hart y Cook (1992a) señalan que los pacientes con dolor crónico que presentan mayor grado de represión emocional son aquellos con mayor nivel de neuroticismo, manifestando a su vez un alto grado de depresión, ansiedad, hostilidad y vulnerabilidad.

Son más escasos los trabajos recientes que analizan la influencia de la variable extraversión, en el contexto del dolor crónico, sobre la utilización de unas u otras estrategias de afrontamiento y la consecuente adaptación del individuo. Sin embargo, ya Eysenck, en la década de los sesenta (Lynn y Eysenck, 1961), defendía que los sujetos extrovertidos presentan un umbral de dolor más alto que los introvertidos y toleran mejor el dolor. Además, postuló que los extrovertidos expuestos a una situación de dolor prolongado consiguen una mejor adaptación que los sujetos introvertidos. Más recientemente, Ziesat y Gentry (1978) realizan un trabajo en el que participa una muestra de sujetos con dolor crónico benigno, cuyas conclusiones apuntan que los sujetos con altos 
niveles de introversión manifiestan mayor nivel de dolor percibido, resultado coincidente con un estudio empírico llevado a cabo por la autora de este trabajo (RamírezMaestre, Valdivia, Esteve y López, 2000). Por otro lado, Wade y colaboradores (1992b) señalan que la extraversión influye negativamente en los niveles de malestar subjetivo relacionado con el dolor, aunque no se relaciona con la intensidad percibida de dolor. Posteriormente, Phillips y Gatchel (2000) defienden que las características del sujeto extrovertido (expresa sus sentimientos, es activo socialmente y recibe apoyo social) les lleva a utilizar una serie de estrategias que permiten al paciente conseguir una menor intensidad percibida de dolor. Finalmente, como señalan Phillips y Gatchel (2000), la conclusión general a la que llegan los distintos trabajos de investigación acerca del efecto de la extraversión sobre el dolor crónico es que los sujetos con altos niveles de extraversión son los que muestran un umbral de dolor más alto y mayor tolerancia al dolor. A pesar del limitado número de trabajos que analizan la relación entre los niveles de extraversión y las estrategias de afrontamiento utilizadas por pacientes que padecen dolor crónico, los estudios acerca del papel de la extraversión en los sujetos sometidos a situaciones estresantes subrayan la necesidad de indagar en este sentido, esperándose resultados similares en el ámbito específico del dolor crónico.

\section{OPTIMISMO DISPOSICIONAL}

Nos referimos al optimismo disposicional como la tendencia a mantener expectativas positivas sobre el futuro (Garofalo, 2000). Los trabajos de Scheier y Carver $(1985,1992)$ muestran que esta tendencia a anticipar resultados positivos es relativamente estable a través del tiempo y las distintas situaciones. A menudo se explica el optimismo disposicional como un modelo de autorregulación cuya efectividad depende del grado de congruencia entre la expectativa y la realidad. Esto es, unas expectativas positivas unidas a una evaluación realista de los acontecimientos futuros puede llevar al sujeto a conductas que aumentan las posibilidades de éxito (Garofalo, 2000). Hay un creciente número de estudios que apoyan los efectos positivos del optimismo sobre la salud y el bienestar psicológico (Affleck y Tennen, 1996; Carver, Pozo, Harris, Noriega, Scheier, y cols., 1993, 1994; Fitzgerald, Tennen, Affleck y Pransky, 1993: Jenkins, 1996; Nelson, Carr y Coleman, 1995; Scheier y Carver, 1987; 1992; Scheier, Matthews, Qwens, Magovern, Lefebvre y cols., 1989). Parece que las expectativas positivas pueden apartar al paciente de las consecuencias psicológicas negativas que acompañan a la enfermedad.
Esta protección lleva a una mejora general de la adaptación del sujeto a la situación estresante a la que se ve sometido (Carver y cols., 1993; Taylor, 1983). Posiblemente, esta buena adaptación del individuo venga mediada por las estrategias de afrontamiento utilizadas, de forma que el optimista hará uso de estrategias más efectivas que el pesimista. Carver, Scheier y Weintraub (1989) señalan que los optimistas confían más en las estrategias centradas en el problema que los pesimistas, los cuales utilizan en mayor medida las estrategias de afrontamiento centradas en la emoción.

En el ámbito del dolor, algunos trabajos señalan que una disposición optimista puede conducir a un tratamiento exitoso en sujetos con dolor (Gruen, 1972; Haerkaepaeae, Jaervikovski, y Estlander, 1996; Jaminson, Taft, O'Hara y Ferrante, 1993; Novy, Nelson, Hetzel, Squitieri y Kennington, 1998). De hecho, uno de los objetivos de la intervención psicológica en sujetos con dolor crónico ha sido conseguir la reducción del catastrofismo como estrategia de afrontamiento, lo cual suele llevar consigo una reducción de la intensidad de dolor percibido y una mejor adaptación del paciente a la situación (Turner y Clancy, 1986). Los escasos estudios realizados acerca del optimismo y la experiencia de dolor, señalan la existencia de efectos positivos del primero sobre el segundo. Así, Haerkaepaeae y colaboradores (1996) evaluaron la capacidad de los factores psicológicos para predecir los resultados de un programa multidisciplinar de tratamiento del dolor de espalda. El estudio muestra que el optimismo y las creencias de control predicen dichos resultados. Parece que los pacientes más optimistas y con mayor percepción de control muestran mejoras superiores a largo plazo después del tratamiento. Además es más probable que los pacientes optimistas retomen sus puestos de trabajo.

Otro estudio empírico a este respecto es el realizado por Long y Sangster (1993) acerca del papel del optimismo disposicional en las estrategias de afrontamiento utilizadas por una muestra de pacientes con dolor crónico. Los resultados muestran que los pacientes más optimistas utilizan en mayor medida estrategias de solución de problema. Aunque los datos encontrados no indican una asociación entre las estrategias de solución de problemas y la adaptación a la situación de dolor, si muestran una relación entre la disposición optimista y una mejor adaptación psicológica. En congruencia con estos resultados, Novy y colaboradores (1998) observaron que altos niveles de optimismo se relacionan con la utilización de estrategias de afrontamiento activas en sujetos con dolor crónico. A su vez, estas estrategias se relacionan con la percepción de control.

En definitiva, como señalan Scheier y Carver (1992), 
altos niveles de optimismo llevan al paciente a llevar a cabo un afrontamiento activo y centrado en el problema. Como ya se ha señalado en líneas anteriores, este tipo de afrontamiento se ha relacionado con un funcionamiento más adaptativo, una reducción de las secuelas psicológicas y una mejora de los resultados de los tratamientos (Brown y Nicassio, 1987; Fernández y Turk, 1989; Turk, 1996).

En definitiva, el estudio de las diferencias entre individuos en función de variables personales tan definitivas como las aquí estudiadas, se presenta como un campo de análisis apasionante en el ámbito del estrés y, más concretamente del dolor crónico. Nos encontramos inmersos en un debate propiciado por el amplio número de datos, en muchas ocasiones contradictorios, respecto a la influencia de dichas características personales en la percepción e informe que realiza el paciente con dolor crónico acerca de su experiencia dolorosa, así como de las distintas acciones y pensamientos que lleva a cabo el individuo para hacer frente a la situación estresante a la que se ve sometido. Esperamos que futuros trabajos continúen en esta línea, arrojando luz sobre las incógnitas planteadas.

\section{REFERENCIAS}

Affleck, G., Tennen, H., Urrows, S., y Higgins, P. (1992). Neuroticism and the pain-mood relation in rheumatoid arthritis: Insights from a prospective daily study. Journal of Consulting and Clinical Psychology, 60, 119-126.

Affleck, G. y Tennen, H. (1996). Construing benefits from adversity: Adaptational significance and dispositional underpinnings. Journal of Personality, 64, 899-922.

Affleck, G., Tennen, H., Keefe, F.J., Lefevre, K. y cols. (1999). Everyday life with osteoarthritis of rheumatoid arthritis: Independent effects of disease and gender on daily pain, mood and coping. Pain, 83, 601-609.

Asghari, M.A. (1997). The relative contribution of personality and pain related beliefs /coping strategies in adjustment to chronic pain. Dissertation Abstrac International Section B: The Sciences and Engineering, 58, 1586.

Asghari, M. A., y Nicholas, M. K. (1999). Personality and adjustment to chronic pain. Pain Reviews, 6, 85-97.

Astor-Dubin, L. y Hammen, C. (1984). Cognitive versus behavioral coping response of men and women: a brief report. Cognitive Therapy and Research, 85-90.

Backman, L. y Molander, B. (1986a). Adult age differences in the ability to cope with situations of high arousal in precision sport. Psychology and Aging, 1, 133-139.

Backman, L. y Molander, B. (1986b). Effects of adult age and level of skill on the ability to cope with high-stress conditions in a precision sport. Psychology and Aging, 1, 334-336.

Backman, L. y Molander, B. (1991). On the generalizability of the age-related declined in coping with high arousal conditions in a precision sport: Replication and extension. Journal of Gerontology, 46, 79-81.
Beecher, H.K. (1965). Quantification of the subjetive pain experience. En M. Weisenberg (Ed.). Pain. New York: The C.V. Mosby Company.

BenDebba, M; Torgerson, W.S. y Long, D.M. (1997). Personality traits, pain duration and severity, functional impairment and psychological disstres in patient with persistent low back pain. Pain, 72, 115-125.

Birren, J.E., Shapiro, H.B., y Miller, H.H. (1959). The effect of salicylate upon pain sensitivity. Journal of Pharmacology and Experimental Therapeutics, 100, 67-71.

Bolger, N. (1990). Coping as a personality process: A prospective study. Journal of Personality and Social Psychology, 59, 525-537.

Bonica, J.J. (1953). The management of pain. Filadelfia: LEA.

Brems, C. y Johnson, M.E. (1989). Problem-solving appraisal and coping style: The influence of sex-role orientation and gender. Journal Psychology, 123, 187-194.

Brown, G. K., y Nicassio, P. M. (1987). The development of a questionnaire for the assessment of active and passive coping strategies in chronic pain patients. Pain, 31, 53-65.

Buckelew, S.P., Shutty, M.S., Hewett, J., Landon, T., y cols. (1990). Health locus of control, gender differences and adjustment to persistent pain. Pain, 42, 287-294. Carver, C.S., Pozo, C., Harris, S.D., Noriega, V., Scheier, M.F., Robinson, D.S., Ketcham, A.S., Moffat, F.L., y Clark, K.C. (1993). How coping mediates the effect of optimism on distress: A study of women with early stage breast cancer. Journal of Personality and Social Psychology, 65, 375-390.

Carver, C.S., Pozo, C., Harris, S.D., Noriega, V., Scheier, M.F., Robinson, D.S., Ketcham, A.S., Moffat, F.L., y Clark, K.C. (1994). Optimism versus pessimism predicts the quality of women's adjustment to early stage breast cancer. Cancer, 73, 1213-1320.

Carver, C.S., Scheier, M.F. y Weintraub, J.K. (1989). Assessing coping strategies: A theoretically based approach. Journal of Personality and Social Psychology, 56, 267-283.

Clark, W.C. y Meehl, L. (1973). Signal detection theory procedures are not equivalent when termal stimulis are judged. Journal of Experimental Psychology, 97, 48-53.

Collin, G. y Stone, L.A. (1966). Pain sensitivity, age and activity level in chronic schizofrenics and in normals. British Journal of Psychiatry, 112, 33-55.

Cornelius, S.W. y Caspi, A. (1987). Everyday problem solving in adulthood and old age.Psychology and Aging, 2, 144-153.

Costa, P. T., Jr., y McCrae, R. R. (1990). Personality: Another "hidden factor" in stress research. Psychological Inquiry, 1, 2224.

Costa, P.T., Zonderman, A.B., y McCrae, R.R. (1991). Personality, defense, coping, and adaptation in older adulthood. En A.L.Greene y K.H. Karraher (Eds.). Life-Span developmental psychology: Perspectives on stress and coping. Hillsdale, New Jersey: Erlbaum.

Costa, P.T. y McCrae, R.R. (1993). Psychological stress and coping in old age. En L. Goldberger y S. Breznitz (Eds.). Handbook of stress: Theoretical and clinical aspects. New York: Free Press.

Costa, P.T., Sommerfield, M.R ., y McCrae, R.R. (1996). Personality and coping: A reconceptualization. En M. Zeidner y N.S. Endler (Eds.). Handbook of Coping: Theoretical, Research, Applications. New York: Wiley.

Crook, J., Rideout, E. y Browne, G. (1984). The prevalence of pain complaints in a general population. Pain, 18, 299-314.

Cruzado, J.A., Labrador, F.J., De la Puente, M.L. y Vallejo, M.A.
ANÁLISIS 
(1990). En F. Fuentenebro, y C. Vázquez (Eds.). Psicología Médica, Psicopatología y Psiquiatría. Madrid: McGraw-Hill.

David, J.P. y Suls, J. (1999). Coping efforts in daily life: Role of Big Five traits and problem appraisals. Journal of Personality, 67, 265-294.

Dejong, G.M., Van Sonderen, E., y Emmelkamp, P.M.G. (1999). A comprehensive model of stress: The roles of experienced stress and neuroticsm in explaining the stress-distress relationship. Psychotherapy and Psychosomatics, 68, 290-298.

Donker, F.J.S. (1991). Evaluación y tratamiento conductual del dolor crónico. En G. Buela-Casal y V.E. Caballo (Comps.). Manual de Psicología Clinica Aplicada. Madrid: Siglo XXI.

Ellermeier, W. y Westphal, W. (1995). Gender differences in pain ratings and pupil reactions to painfull presure stimuli. Pain, 61, 435-439.

Endler, N. S., y Parker, J. D. A. (1990). Multidimensional assessment of coping: A critical evaluation. Journal of Personality and Social Psychology, 58, 844-854.

Epstein, S., y Meier, P. (1989).Constructive thinking: A broad coping variable with specific components. Journal of Personality and Social Psychology, 57, 332-350.

Faucett, J., Gordon, N., y Levine, J. (1994). Differences in postoperative pain severity among four ethnic groups. Journal of Pain and Symptom Management, 9, 383-389.

Feifel, H., Strack, S. Y Nagy, V.T. (1987). Coping strategies and associated features of medically ill patients. Psychosomatic Medicine, 49, 616-625.

Feine, J.S., Bushnell, M.C., Miren, D., y Duncan, G.H. (1991). Sex differences in the perception of noxious heat stimuli. Pain, 44, 255-262.

Felton, B.J. y Revenson, T.A. (1987). Age differences in coping with chronic illness. Psychology and Aging, 2, 164-170.

Fernández, E., y Turk, D.C. (1989). Utility of cognitive coping strategies for altering perceptions of pain: A meta-analysis. Pain, 38, 123-135.

Fitzgerald, T., Tennen, H., Affleck, G., y Pransky, G. (1993). Relative importance of dispositional optimism and control appraisals in quality of life after coronary artery bypass surgery. Journal of Behavioral Medicine, 16, 25-43.

Flor, H. Y Turk, D.C. (1984). Ethiological theories and treatment for chronic back pain I. Somatic models and interventions. Pain, 19, 105-121.

Folkman, S., Lazarus, R., Pimley, S. y Novacek, J. (1987). Age differences in stress and coping processes. Psychology and Aging, 2, 171-184.

Fordyce, W.E. (1976). Behavioral methods for chronic pain and illness. San Luís: The C.V. Mosby Company.

Gagliese, L., y Melzack, R. (1997). The assessment of pain in the elderly. En D.I.

Motofsky y J. Lomranz, Handbook of Pain and Aging. New York: Plenum Press.

Garofalo, J.P. (2000). Perceived optimism and chronic pain. En R.J. Gatchel y J.N. Weisberg (Eds.), Personality Characteristics of Patients with Pain. Washington: APA.

Gómez, R., Holmberg, K., Bounds, J., Fullarton, C., y Gómez, A. (1999). Neuroticism and extroversion as predictors of coping styles during early adolescence. Personality and Individual Differences, 27, 3-17.

Gruen, W. (1972). Successful application of systematic self-relaxation and self-suggestions about postoperative reactions in a case of cardiac surgery. International Journal of Clinical and Experimental Hypnosis, 20, 143-151.

Gunther, K.C., Cohen, L.H., y Armeli, S. (1999). The role of neuroticism in daily stress and coping. Journal of Personality and Social Psychology, 77, 1087-1100.

Gutman, D.L. (1964). An exploration of ego configurations in middle and later life. En B.L. Neugarten (Ed.). Personality in Middle and Later Life. New York: Atherton.

Gutman, D.L. (1974). The country of old men: Cross-cultural studies in the psychology of later life. En R.A. Levine (Ed.). Culture and Personality: Contemporary Readings. Chicago: Aldine.

Gutman, D.L. (1987). Reclaimed Powers: Toward a Psychology of Men and Women in Later Life. New York: Basic Books.

Haerkaepaeae, K., Jaervikovski, A., y Estlander, A.M. (1996). Health optimism and control beliefs as predictors for treatment outcome of a multimodal back treatment program. Psychology and Health, 12, 123-134.

Harkins, S.W. y Chapman, C.R. (1976). Detection and decision factors in pain perception in young and elderly men. Pain, 2, 253-264.

Harkins, S.W. y Chapman, C.R. (1977). Age and sex differences in pain perception. En B. Andros y B. Mathaws (Eds.). Pain in Trigeminal Regions. North Holland, Amsterdam: Elsevier.

Harkins, S.W. y Price, D.D. (1992). Assessment of pain in the elderly. En D.C. Turk y R. Melzack (Eds.). Handbook of Pain Assessment. New York: Guilford Press.

Helkimo, M. (1979). Epidemiological surveys of dysfunction of the masticatory system. En G.A. Zarb y G.E. Carlsson (Eds.). Temporomandibular joint: Function and dysfunction. Copenhagen: Munkskaard.

Hewitt, P. L. y Flett, G.L. (1996). Personality traits and the coping process. En M. Zeidner y N. S. Endler (Eds.). Handbook of Coping. New York: Wiley.

Holm, J.E., Holroyd, K.A., Hursey, K.G. y Penzien, D.B. (1986). The role of stress in recurrent tension headache. Headache, 26, 160-167.

International Association for the Study of Pain (1986). Classification of chronic pain. Pain, Suppl. 3, 19-32.

Irion, J.C. y Blanchard-Fields, F. (1987). A cross-sectional comparison of adaptive coping in adulthood. Journal of Gerontology, 42, 502-504.

Jamison, R.N., Taft, K., O’Hara, J.P., y Ferrante, F.M. (1993). Psychosocial and pharmacologic predictors of satisfaction with intravenous patient-controlled analgesia. Anesthesia Analgesia, 77, 121-125.

Jelicic, M.; Kempen, G.I.J.M. y Passchier, J. (1998). Psychological well-being in older adults suffering from chronic headache. Headache, 38, 292-294.

Jenkins, C.D. (1996). While there's hope, there's life. Psychosomatic Medicine, 58, 122-124.

Jensen, M.P., Turner, J.A., Romano, J.M., y Lawler, B.K. (1994). Relationship of pain specific beliefs to chronic pain adjustment. Pain, 57, 301-309.

Kanner, A.D., Coyne, J.C., Schaefer, C. y Lazarus, R.S. (1981). Comparison of two modes of stress measurement: Daily hassles and uplifts versus major life events. Journal of Behavioral Medicine, 4, 1-39.

Keefe, F.J. y Williams, D.A. (1990). A comparison of coping strategies in chronic pain patiens in different age groups. Journal of Gerontology, 45, 4, 161-165.

Keefe, F.J., Crisson, J., Urban, B.J., y Williams, D.A. (1990). Analyzing chronic low back pain: The relative contribution of pain coping strategies. Pain, 40, 293-301.

Kempen, G.I.J.M.; Jelicic, M. y Ormel, J. (1997). Personality, chronic medical morbidity, and health related quality of life 
among older persons. Health Psychology, 16, 539-546.

Khrone, H.W., Schumacher A., y Egloff, B. (1992). Das Angstbewaltigungs - Inventar (AIB). Mainz, Germany: Johanes Gutenberg-Unversitat, Psychologisches Institut.

Klinger, L. y Spaulding, S.J. (1998). Chronic pain in the elderly: Is silence really golden?. Physical and Occupational Therapy in Geriatrics, 15, 1-17.

Krohne, H.W. (1996). Individual differences in coping. En M. Zeidner y N.S. Endler (Eds.).Handbook of Coping: Theoretical, Research, Applications. New York: Wiley.

Labouvie-Vief, G., Hakim-Larson, J., y Hobart, C.J. (1987). Age, ego level, and the life-span development of coping and defense processes. Psychology and Aging, 2, 286-293.

Labrador, F.J. y Vallejo, M.A. (1984). Dolor crónico. En J. Mayor y F.J. Labrador (Eds.). Manual de Modificación de Conducta.Madrid: Alhambra.

Lauver, S.C. y Johnsosn, J.L. (1997). The role of neuroticism and social support in older adults with chronic pain behavior. Personality and Individual Differences, 23, 165-167.

Lazarus, R. S., y Folkman, S. (1984/1986). Estrés y procesos cognitivos. Barcelona: Martínez Roca.

Lazarus, R.S. (1993). Coping theory and research: Past, present and future. Psychosomatic Medicine, 55, 237-247.

Lefebvre, J.C. (1998). The effects of neuroticism and induced affect on the recall of pain intensity, pain unpleasantness and perceived daily activity interference in chronic low back pain. Dissertation Abstract International Section B: The Sciences and Engineering, 59, 1859.

Linton, S.J. (1987). Chronic pain: The case for prevention. Behavior Research and Therapy, 313-317.

Lipton, J.A., Ship, J.A., y Larach-Robinson, D. (in press). Prevalence reported jaw, joint, face and burning mouth pain in the United States. Journal of Am. Dent. Assoc.

Long, B.C., y Sangster, J.I. (1993). Dispositional optimism/ pessimism and coping strategies: Predictors of psychosocial adjustment of rheumatoid and osteoarthritis patients. Journal of Applied and Social Psychology, 23, 1069-1091.

Lynn R. y Eysenck, H.J. (1961). Tolerance for pain, extraversion and neuroticism. Perceptual and Motor Skills, 12, 161162Madrid-Arias, J.L. (1987). Clínicas del dolor intolerable. En F.J. Florez (Dir.). Dolor. Madrid: IDEPSA.

Margolis, R.B., Zimmy, G.H., Miller, D. Y Taylor, J.M. (1984). Internist and the chronic pain patients. Pain, 20, 151-156.

Martin, M.Y., Bradley, L.A., Alexander, R.W., Alarcón, G.S., Triana-Alexander, M., Aaron, L.A., y Alberts, K.R. (1996). Coping strategies predict disability in patients with primary fibromyalgia. Pain, 68, 45-53.

McCrae, R.R. (1982). Age differences in the use of coping mechanisms. Journal of Gerontology, 37, 454-460.

McCrae, R.R. y Costa, P.T. (1986) Personality, coping, and coping effectiveness in an adult sample. Journal of Personality, 54, 385-405.

McCrae, R.R. y Costa, P.T. (1990). Personality in Adulthood. New York: Guilford.

Medvedova, L. (1998). Personality dimensions - "Little Five"and their relationships with coping strategies in early adolescence. Studia Psychologica, 40, 261-265.

Medvedova, L. (1999). Personality factors and coping with stress in pubescents. Psychologia a Pathopsychologia Dietata, 34, 312

Miller, S.M. y Kirsch, N. (1987). Sex differences in cognitive coping with stress. En R.C. Barnett, L. Biener y G.K. Baruch
(Eds.). Gender and Stress. New York: Free Press.

Miró, E.y Buela-Casal, G. (1996). Evaluación del dolor crónico. En G. Buela-Casal, V. Caballo y J.C. Sierra (dirs.). Manual de Evaluación en Psicología Clínica y de la Salud. Madrid: Siglo XXI.

Mobily, P., Herr, K., Clark, M. y Wallace, R. (1994). An epidemiologic analysis of pain in the elderly: The Iowa 65+ rural health study. Journal of Aging and Health, 6, 139-154.

Morasso, G., Costantini, M., Baracco, G., Borreani, C., y Capelli, M. (1996). Assessing psychological distress in cancer patients: Validation of a self-administered questionnaire. Oncology, 53, 295-302.

Nelson, E.S., Karr, K.M., y Coleman, P.K. (1995). Relationships among daily hassles, optimism and reported physical symptoms. Journal of College Student Psychotherapy, 10, 1126.Novy, D.M., Nelson, D.V., Hetzel, R.D., Squitieri, P., Kennington, M. (1998). Coping with chronic pain: Sources of intrinsic and contextual variability. Journal of Behavioral Medicine, 21, 19-34.

Oleson, J. (1978). Some clinical features of the acute migraine attack: An analysis of 750 patients. Headache, 16, 265-271.

Pearlin, L.I. y Schooler, C. (1978). The structure of coping. Journal of Health and Social Behavior, 19, 2-21.

Pfeiffer, E. (1977). Psychopatology and social pathology. J.E. Birren y K.W. Schaie (Eds.). Handbook of the Psychology of Aging. New York: Van Nostrand Reinhold.

Phillips, J.M. y Gatchel, R.J. (2000). Extraversion - Introversion and chronic pain. En R.J. Gatchel y J.N. Weisberg (Eds.). Personality Characteristics of Patients with Chronic Pain. Washington: APA.

Porter, A., y Stone, A.A. (1995). Are there really gender differences in coping? Journal of Social and Clinical Psychology, 14, 15-22.

Prohaska, T.R., Leventhal, E.A., Leventhal, H. y Keller, M.L. (1985). Health practices and illness condition in young, middle aged, and elderly adults. Journal of Gerontology, 40, 569-578.

Quayhagen, M.P., y Quayhagen, M. (1982). Coping with conflict. Research on Aging, 4, 364-377.

Ramírez-Maestre, C.; López, A.E. y Anarte, M.T. (1999). Influencias de las variables sexo y edad en las estrategias de afrontamiento y el dolor crónico. Comunicación presentada a la IV Jornada de la SEIDI. Valencia.

Ramírez-Maestre, C., Valdivia, Y., Esteve, R., y López (2000). Neuroticismo, afrontamiento y dolor crónico. I Congreso Hispano-Portugués de Psicología. Santiago de Compostela.

Reid, G.J., Gilbert, C.A. y McGrath, P.J. (1994). Development of a pain coping questionnaire. Third International Symposium on Pediatric Pain. Filadelfia.

Rim, Y. (1986). Ways of coping, personality, age, sex, and family structural variables. Personality and Individual Differences, $7,133-116$.

Rollman, G.B. y Harris, G. (1987). Detectability, discriminability, and perceived magnitud of painful electric shock. Perceptual Psychophysiology, 42, 257-268.

Ruda, M.A. (1993). Gender and Pain. Pain, 63, 1-2.

Sánchez Cánovas, J. y Sánchez López, M.P. (1994). Psicología diferencial: Diversidad e individualidad humanas. Madrid: Ramón Areces.

Scheier, M.F., y Carver, C.S. (1985). Optimism, coping and health: Assessment and implications on generalized outcome expectancies. Health Psychology, 4, 219-247.

Scheier, M.F., y Carver, C.S. (1987). Dispositional optimism 
and physical well-being: The influence of generalized outcome expectancies on health. Journal of Personality, 55, 169210.

Scheier, M.F., y Carver, C.S. (1992). Effects of optimism on psychological and physical well-being: Theoretical overview and empirical update. Cognitive Therapy and Research, 16, 201228.

Scheier, M.F., Matthews, K., Qwens, J., Magovern, G., Lefebvre, R., Abbot, R., y Carver, C.S. (1989). Dispositional optimism and recovery from coronary artery bypass surgery: The benefitial effects on physical and psychological well-being. Journal of Personality and Social Psychology, 57, 1024-1040.
Turk, D.C. y Okifuji, A. (1999). Does sex make a difference in the prescription of treatments and the adaptation to chronic pain by cancer and non-cancer patients?. Pain, 82, 139-148.

Turner, J. A. y Clancy, S. (1986). Strategies for coping with chronic low back pain: Relationships to pain and disability. Pain, 24, 355-363.

Unruh, A.M. (1996). Gender variations in clinical pain experience. Pain, 65, 123-167.

Vaillant, G.L. (1976). Natural history of male psychological health: 5 . The relation of choice of ego mechanims of defense to adult adjustment. Archives of General Psychiatry, 33, 535-545.

Vaillant, G.L. (1977). Adaptation of Life. Boston: Little, Brown.

Verbrugge, L.M. (1985). Gender and health: an update on hypotheses and evidence. Journal of Health and Social Behavior, 26, 156-182.

Vingerhoets, A.J. y Van Heck, G.L. (1990). Gender, coping and psychosomatic symptoms. Psychological Medicine, 20, 125135.

Von Korff, M., Dworkin, S.F., y Kruger, A. (1988). An epidemiologic comparison of pain complaints. Pain, 32, 173-183.

Wade, J. B., Dougherty, L. M., Hart, R.P., y Cook, D.B. (1992 a). Patterns of normal personality structure among chronic pain patients. Pain, 48, 37-43.

Wade J. B., Dougherty, L. M., Hart, R.P., y Rafii, A. (1992b). A canonical correlation analysis of the influence of neuroticism and extraversion on chronic pain, suffering and pain behavior. Pain, 51, 67-73.

Weidner, G. Y Collins, R.L. (1993). Gender, coping and health. En H.W. Khrone (Ed.). Attention and avoidance. Strategies in coping with aversiveness. Seattle: Hogrefe y Huber.

Ziesat, H.A., Jr., y Gentry, W.D. (1978). Pain Aperception Test: An investigation of concurrent validity. Journal of Clinical Psychology, 34, 786-789.

Zimbardo, P.G., Cohen, A.R., Weisenberg, M., Dwokin, L. y Firestone, I. (1966). Control of pain motivation by cognitive dissonance. Science, 151, 217-219.

Zoob, M. (1978). Differentiating the chest pain. Geriatrics, 33,

\footnotetext{
95-101. ment.Nueva York: Guilford Press.
}

Turk, D.C. y Melzack, R. (1992). Handbook of Pain Assess-

Turk, D.C. (1996). Biopsychosocial perspective on chronic pain. En R.J. Gatchel y D.C. Turk (Eds.), Psychological approaches to pain management. Nueva York: Guilford Press. 